\title{
Calcul mécanique des lignes électriques dans le cas de longues portées en forte pente \\ (SUITE IT FIN) (1)
}

Dans la première partie de cette élade, l'auteur avait élabli les formules mathématiques permettant de déterminer, dans tous les cas, d'une manière rigoureuse, les conditions de pose d'un câble Dans le présent article, il examine d'abord les solutions pratiques qui ont fait l'objet de publications dans d'autres périodiques, puis il expose la méthode de similitude employée par lui; des exemples numériques permettent de comparer ces divers procédés.

\section{II. -- Méthodes pratiques de calcul}

1. La méthode de la Verband deutsche El. $\left({ }^{2}\right)$, est basée sur les propriétés ci-après de la chaînette, approximatives mais suffisamment exactes dans le cas des portées inclinées habituelles :

a) Le lieu des milieux des cordes parallèles à une direction fixe est une droite verticale passant par le point de contact de la tangente parallèle à cette direction.

b) Dans les formules (1) et (2) (1) donnant $Y$ et $l$ en fonction de $x$, on peut négliger $\left(\frac{x}{m}\right)^{\mathrm{r}}$ à partir de la puissance trois.

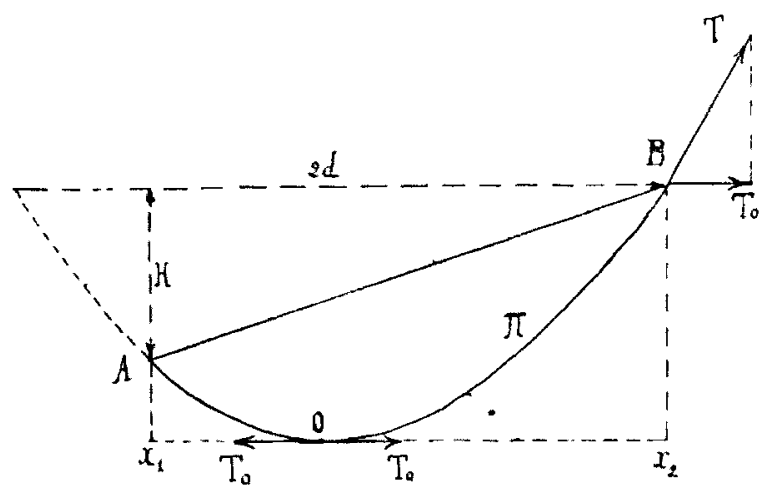

Fig. 1

c) La tension moyenne $T_{m}$ de la portée inclinée $A B$ est la tension au point de contact de la tangente parallèle à $\mathrm{A} B$.

Il en résulte alors, pour la flèche la valeur :

$$
f=\frac{y_{1}+y_{2}}{2}-y_{\mathrm{m}}
$$

(1) Voir la Houille Blanche de mai-juin 1926.

(2) Voir la R. G. E. du 10 mai 1924. expression dans laquelle $y_{\mathrm{m}}$ est l'ordonnée du point de contact dont l'abscisse est considérée comme égale à

$$
\frac{x_{2}+x_{2}}{2} \text {. }
$$

L'équation (1) (') pe:met d'exprimer $y_{1} y_{2}$ en fonction de $x_{1} x_{2}$ et donne aussi :

$$
y_{\mathrm{m}}=m \cos h\left(\frac{1}{m} \cdot \frac{x_{1}+x_{2}}{2}\right)-m
$$

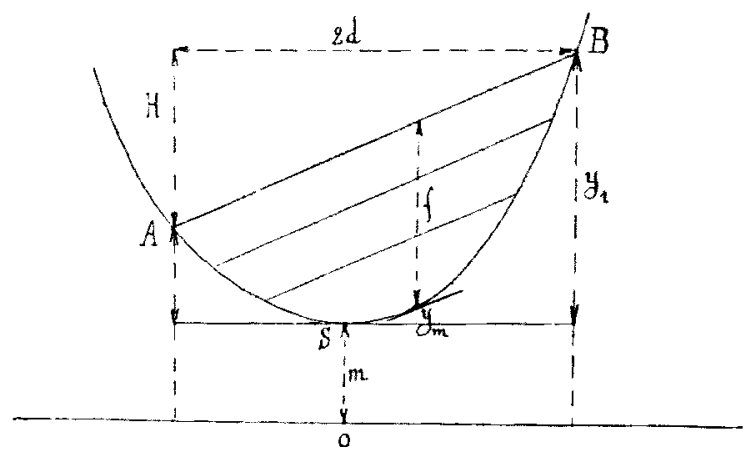

Fig. 2

Par des transformations successives et en négligeant les puissances de $\frac{d}{m}$ supérieurs à quatre, on trouve, en portant ces expressions de $y_{1} y_{2} y_{\mathrm{m}}$ dans celle de $f$ :

$$
f=\frac{(2 d)^{2}}{8 m} \cos h\left(\frac{1}{m} \cdot \frac{x_{1}+x_{2}}{2}\right)
$$

La formule (8) (1), devient, d'autre part, un négligeant les puissances de $\frac{d}{m}$ supérieures à ́rois :

$$
H=2 d \sin h\left(\begin{array}{cc}
1 & x_{1}+\ddot{z} \\
m & \cdots
\end{array}\right)
$$

(1) Voir la Houille Blanche de :.: i-juin 1926.

\section{ERE ATUTM}

No 117-118, Septembre-Octobre 1926: Compensaleurs de phase pour Moleurs asynchrones.

Page 153, ge formule, lire :

$$
\frac{\omega^{\prime} t}{120} \text { au lieu de } \frac{\omega^{\prime} i}{120}
$$

Page 153, colonne de droite, $4^{0}$ ligne après la figure, lire $B_{3} B_{1}$ au lieu de $B_{1} B_{2}$

Page 153, colonne de droile, 17 e ligne après !' finure, lire : $e_{1}^{\prime} e_{2}^{\prime \prime}$ au lieu de $e_{3}^{\prime} e_{2}^{\prime \prime}$

Page 15', colonne de gauche, dans les formules, $P=\ldots, Q=\ldots$, lire: 
En élevant au carré ces deux équations et les retranchant ensuite membre à membre :

$$
f^{2}=\frac{(2 d)^{2}}{64 m^{2}} \cdot\left(1+\frac{H^{2}}{(2 d)^{2}}\right)
$$

On se rappelle que le module $m$ représente $\frac{T_{0}}{I I}, T_{0}$ étant la tension au point $\mathrm{S}$ et II la force externe appliquée au câble par unité de longueur.

De l'hỵpothèse (a) résulte :

$$
\mathrm{T}_{0}=\mathrm{T}_{\mathrm{m}} \cos \mathrm{p}=\mathrm{T}_{\mathrm{m}} \cdot \frac{2 d}{\mathrm{AB}}
$$

et, par suite :

$$
f=\frac{\Pi \cdot \overline{\mathrm{AB}}^{2}}{8 \mathrm{~T}_{\mathrm{n}}}
$$

Enfin, de l'équation (2), par des transformations analogues aux précédentes et avec les mèmes approximations, on tire, pour la longueur " $l$ " de l'arc de la chaînette A B :

$$
l=\mathrm{AB}+\frac{1}{24} \cdot \frac{\Pi^{2} \overline{\mathrm{AB}}}{\mathrm{T}_{\mathrm{m}}^{2}}
$$

Les formules (12) et (13) étant de la même forme que celles ci-après relatives aux chaînettes horizontales

$$
f_{0}=\frac{\pi \cdot(2 d)^{2}}{8 \mathrm{~T}_{0}} \quad l_{0}=2 d+\frac{1}{2 t} \cdot \frac{\pi^{2}(2 d)^{3}}{\mathrm{~T}_{0}{ }^{2}}
$$

représentées par les abaques de $M$. Blondel, le calcul de $f$ et

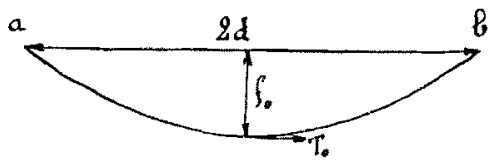

Fig. 3

de $T_{m}$ pourra être effectué au moyen de ces abaques dans lesquels on entrera avec $\mathrm{A} B$ et $\mathrm{T}_{\mathrm{m}}$ au lieu de $2 d$ et $\mathrm{T}_{0}$.

On sait que, en plus des approximations précédemment énumérées, l'emploi de ces abaques en implique une quatrième qui consiste à négliger le produit

$$
*(l-\mathrm{A} \mathrm{B})
$$

Le calcul de la tension au point le plus haut est ici très simple puisque, par suite des approximations admises :

$$
\mathrm{T}=\mathrm{T}_{\mathrm{m}}+\mathrm{II} f+\mathrm{II} \frac{\mathrm{H}}{\mathrm{q}}
$$

2. M. Bachet (1) préconise un procédé de calcul, applicable à toutes les portées inclinées, qui aboutit aussi à l'utilisation des abaques de $M$. Blondel. Les approximations admises sont les suivantes :

a) Pour une portée fixe et un même câble on peut négliger le carré de la variation de longueur de l'arc AB résultant des variations de $T_{0}$.

b) Les terme $\left(\frac{d}{m}\right)^{p}$ peuvent être remplacés par des coefficients fixes égaux à leur valeur moyenne si « $p$ » est plus grand que

(1) Voir R. G. E. des 15 et 29 mai 1926. quatre, tant que l'amplitude des variations du module se maintient entre certaines limiles.

c) Ja variation de la longueur $l$ d'un câble sans tension, provoquée par une variation $\Delta \theta$ de la température, peut être considérée comme égale à $\alpha l^{\prime} \Delta$ o.

Cette approximation revient à négliger le produit $\Delta l_{0}, \Delta 0$ (en appelant $l_{0}$ la longueur sans tension a $0^{\circ}$ ).

d) La variation de la longueur " $l$ " d'un câble sous tension peut ètre considérée comme la somme algébrique des variations qui résulteraient, séparément, d'une part de $\mathcal{S}^{\mathrm{T}}$, d'autre part de $\Delta \theta$ (cela est d'ailleurs mathématiquement vrai pour des variations infiniment petiles); de sorte que :

$$
\Delta l=l\left(\lambda \Delta \theta+\lambda \Delta \mathrm{T}_{0}\right)
$$

e) La longueur infiniment petite $d l_{0}$ d'un élément de câble au repos peut ètre considérée comme égale, à un infiniment près d'un ordre supérieur et négligeable, à la longueur $d l$ de l'arc élémentaire formé par ce même élément sous tension.

Des approximatives ci-dessus résultent : la lormule :

$$
\Delta l=p_{1}\left(\frac{2 d}{l_{i}}\right)^{2}\left[\frac{\pi^{2}(2 d)^{2}}{2-4 \mathrm{~T}_{0}^{2}}-\frac{l^{2}(2 d)^{2}}{21 \mathrm{~T}^{2}}\right]
$$

dans laquelle $p_{1}$ est un paramètre, fixe tant que $\frac{\| l}{\mathrm{~T}_{0}}$ ne sort pas de certaines limites, " $l_{\mathrm{i}}$ " la longueur initiale, $\mathrm{T}_{\mathrm{i}}$ la tension horizontale initiale, $\mathrm{T}_{\mathbf{0}}$ la tension horizontale finale, et la formule :

$$
\Delta l=x l_{\mathrm{i}} \Delta \theta+v\left[\int_{\mathrm{x}_{1}}^{\mathrm{x}_{z}} t\left(d l_{0}\right)-\int_{\dot{x}_{i}}^{\mathrm{x}_{z}} l_{\mathrm{i}}\left(d l_{0}\right)\right]
$$

dans laquelle " $t$ " est la tension finale en un point quelconqut. $t_{i}$ la tension initiale en ce mème point.

Comme, par suite des propriétés connues de la chainetle:

$$
t=\mathrm{T}_{0} \frac{d l}{d x}, \quad t_{\mathrm{i}}=\mathrm{T}_{\mathrm{i}} \frac{d l_{\mathrm{i}}}{d x}, \quad d l=\cos h\left(\frac{x}{m}\right)
$$

on tire des équations (14) et (15), après une série de transformations :

$$
\text { A. } \Delta \theta=\frac{1}{\alpha}\left[\Delta\left(\frac{\mathrm{B}^{2} \pi^{2}(2 d)^{2}}{2 / 4 \mathrm{~T}_{0}^{2}}\right)-\Delta\left(\frac{\mathrm{T}_{0}}{\mathrm{E}}\right)\right]
$$

où $\mathrm{A}$ et $\mathrm{B}$ sont des constantes à déterminer pour chaque cas particulier.

Cette équation ayant la même forme que celle traduite par les abaques de $M$. Blondel, la détermination de $T_{0}$ pourra ètre effectuée au moyen de ces abaques dans lesquels on entrera, non pas avec $\theta$ et $2 d$, mais avec $\mathrm{A} \theta$ et $\mathrm{B}(2 d)$.

Ayant ainsi déterminé le module $m=\frac{T_{0}}{\text { II }}$ c'est-à-dire la chấnette dont fait partie l'arc correspondant à l'équilibre final, on pourra facilement calculer, soit la tension appelée moyenne dans la méthode précédente, soit la tension au point le plus haut.

3. Le calcul par la méthode de similitude est basé sur la considération suivante comnue :

" Deux chaînettes quelconques de module $m$ et $m^{\prime}$ sont semblables et le rapport de similitude est $\frac{m}{m^{\prime}}$. " 
Donc si on fait coïncider en $\mathrm{S}$ les sommets de toutes les chaînet.tes et si on appelle $m m^{\prime} m^{\prime \prime}$... leurs modules, chaque rayon vecteur donne les relations :

$$
\begin{aligned}
& x^{\prime}=\frac{m^{\prime}}{m} x \quad x^{\prime \prime}=\frac{m^{\prime \prime}}{m} x \quad \ldots \ldots \ldots . \quad \ldots \quad \text { ctc } \\
& y^{\prime}=\frac{m^{\prime}}{m} y \quad y^{\prime \prime}=\frac{m^{\prime \prime}}{m} y \quad \ldots \ldots \ldots \ldots \text { e.c. }
\end{aligned}
$$

On sait, d'autre part, que, en appelant Y Y' Y',.., les longueurs

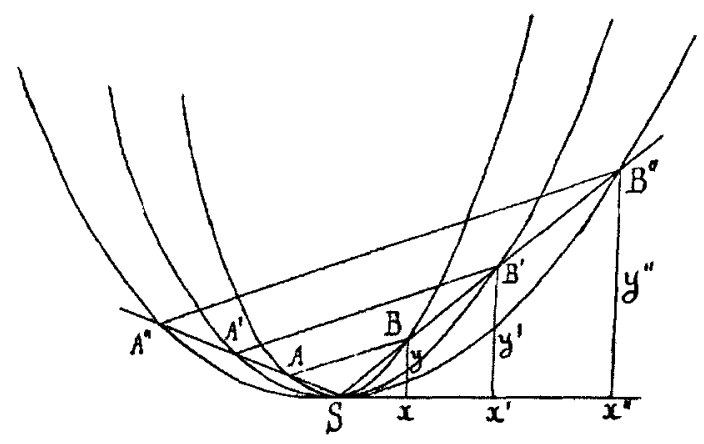

Fig. 4

$y+m, y^{\prime}+m^{\prime}, y^{\prime \prime}+m^{\prime \prime}, \ldots$, les tensions aux points B B' B'... sont II Y II' Y" II" Y"'... :

Comme les deuxièmes relations ci-dessus donnent :

$y^{\prime}+m^{\prime}=\frac{m^{\prime}}{m}(y+m), \quad y^{\prime \prime}+m^{\prime \prime}=\frac{m^{\prime \prime}}{m}(y+m) \ldots$ etc.

On a aussi :

$$
\mathrm{Y}^{\prime}=\frac{m^{\prime}}{m} \mathrm{Y} \quad \mathrm{Y}^{\prime \prime}=\frac{m^{\prime \prime}}{m} \mathrm{Y} \quad \ldots \ldots \quad \text { etc. }
$$

De même en appelant $l_{1} l_{1} l^{\prime \prime}{ }_{1} \ldots$ les longueurs des ar's de chainnette $\$$ B, S B', S B'"... :

$$
l_{1}^{\prime}=\frac{m^{\prime}}{m} l_{1} \quad l_{1}^{\prime \prime}=\frac{m^{\prime \prime}}{m} l_{1} \quad \ldots \quad \ldots \quad \text { etc. }
$$

Ces mêmes relations subsistent pour les longueurs des arcs $\mathrm{SA}, \mathrm{SA}^{\prime} \mathrm{SA} \mathrm{A}^{\prime} . .$. , de sorte que, les longueurs $l l^{\prime} l^{\prime}$ '... des $\operatorname{arcs} \mathrm{AB}$, A' B', A" B"'... sont aussi reliées par :

$$
l^{\prime}:=\frac{m^{\prime}}{m} l \quad l^{\prime}=\frac{m^{\prime \prime}}{m} l \quad \ldots \quad \ldots \text { ete. }
$$

Enfin on sail qu'un câble suspendu entre $P$ et $Q$ est un segment

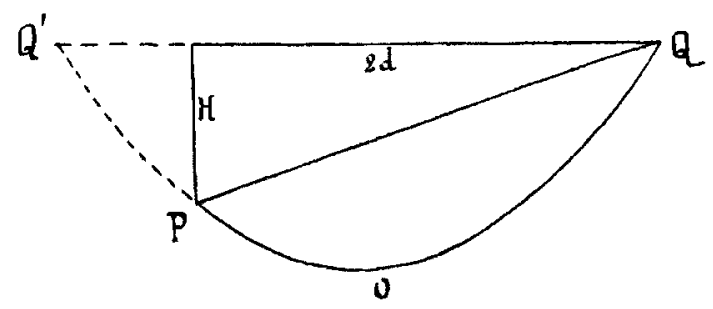

Fig. 5

d'une chainette $Q$ Q' de sommet $o$, de sorte qu'en transportant par la pensée le point o en $S$, le câble vient prendre la place d'une des courbes de la figure 4 , et par suite les relations précédentes permettent d'exprimer les tensions en $P$ et $Q$ ainsi que la longueur de l'arc $P Q$ en fonction des éléments semblables de la chaînette $A B$ dès que l'on connaît le rapport de similitude.

Il suffira dès lors de calculer très exactement tous les éléments géométriques d'une chaînette unique de "référence" de module " $r$ "; ces calculs seront d'ailleurs assez rapides si on utilise des tables hyperboliques d'un emploi courant maintenant. On établira ainsi, au moyen des formules (1) et (2), pour un module $m=r$ :

$1^{\circ}$. Une table $y=f(x)$ pour des intervalles $\Delta x$ suffisamment petits par rapport à $x$.

$2^{\circ}$ Une table des valeurs des arcs " $l_{x}$ " correspondants

$3^{\circ}$ Une table des différences premières : $\hat{\varepsilon}_{\mathbf{x}}=l-x$.

$4^{\circ}$ Une table des rapports $\frac{l_{\mathrm{x}}}{x}$.

Dans la table « $l_{\mathbf{x}}$ ", intercaler les arcs $l_{2}$, soit en les calculant directement ce qui est assez rapide puisque

$$
\sin h \frac{2 x}{r}=2 \sin h \frac{x}{r} \cos h \frac{x}{r}
$$

c'est-à-dire

$$
r \cdot l_{2 \mathrm{x}}=2 l_{\mathrm{x}}(y+r)
$$

soit en les déduisant des valeurs de " $l_{\boldsymbol{x}}$ " voisines au moyen des différences premières et secondes (formule de Newton).

$5^{\circ}$ de la table $l_{\mathrm{x}}$ ainsi complètée on déduit la table de :

$$
\varepsilon_{\mathrm{x}}=l_{2 \mathrm{x}}-2 x
$$

On a ainsi tous les éléments nécessaires pour suivre les variations de la tension du câble $\mathrm{PQ}$ quand les conditions atmosphériques varient; il suffit, en effet, d'utiliser les formules (9), (9bis), (10), (10 bis), dans lesquelles les longueurs $l_{d}, l_{2 d}$, $\Sigma_{d}$, $\varepsilon_{\mathbf{d}}$ sont obtenues en multipliant $l_{\mathrm{x}}, l_{\mathbf{2}}$, $\Xi_{\mathbf{x}}$, ミ2 $\mathrm{x}$, par le rapport de similitude $\frac{m}{r}$.

Soit, par exemple, un câble de poids unitaire $\pi$, de portée $2 d$, dont les points d'appui sont dénivelés de $\mathrm{H}$ et dont on connaît la tension horizontale $T_{\mathbf{i}}$. Il s'agit de déterminer la tension horizontale $T_{0}$ à laquelle sera soumis ce câble, à la même température, quand une surcharge provenant du vent ou du givre donnera lieu, par unité de longueur, à un effort extérieur résultant II.

La longueur initiale du câble sous tension est (voir formule 3) :

$$
\mathrm{L}_{\mathrm{i}}^{2}=\mathrm{H}^{2}+\left(2 l_{\mathrm{d}}\right)^{2}
$$

$2 l_{\mathrm{d}}$ est la longueur d'une chaînette horizontale de module $m_{\mathrm{i}}=\frac{\mathrm{T}_{\mathrm{i}}}{\pi}$ et de portée $2 d$; celle-ci est semblable à la chainette de référence " $r$; l'arc de " $r$ ", semblable au segment $2 l_{\mathrm{d}}^{\mathrm{d}}$, a comme portée

$$
2 x_{\mathrm{i}}=2 d \cdot \frac{r}{m_{\mathrm{i}}}
$$

Comnaissant ainsi $x_{\mathrm{i}}$, les tables précédentes permettent, au moyen de la formule de Newsten, de calculer $l_{x}^{\mathbf{i}}$ et $l_{\underline{x}}^{\mathrm{i}}$. Comme

$$
l_{\mathrm{d}}=l_{\mathrm{x}} \frac{m_{\mathrm{i}}}{r} \quad \text { et } \quad l_{\mathrm{i} \mathrm{d}}=l_{\mathrm{3}} \frac{m_{\mathrm{i}}}{r}
$$

on obtient :

$$
\mathrm{L}_{\mathrm{i}}=\sqrt{\mathrm{H}^{2}+\left(2 l \mathrm{i} \times \frac{m_{\mathrm{i}}}{r}\right)^{2}}
$$


La formule (9) donne alors, pour la tension moyenue initiale:

$$
\mathrm{T}_{\mathrm{m}}^{\mathrm{i}}=\frac{\mathrm{T}_{\mathrm{i}}}{2 \mathrm{~L}_{\mathrm{i}}}\left[2 d+\mathrm{l}_{2 \mathrm{~d}}\left(1+\frac{\mathrm{H}^{2}}{2\left(l_{\mathrm{d}^{2}}\right)^{2}}\right)\right]
$$

La longueur du câble au repos à la même température résulte de la formule $(7)$ :

$$
L_{0}=\frac{L_{i}}{1+\lambda T_{m}}
$$

Lorsque - sera devemu II, le cable sera un segment de chaînette de tension horizontale $\mathrm{T}_{0}$ et de partie $2 d$. J'appelle $2 x$ la projection horizontale du segment semblable de la chainette de référence, $L$ la longueur du càble ainsi surchargé ; on a :

$$
L=V\left(\mathrm{I}^{2}+(2 / \mathrm{d})^{2}\right.
$$

où $2 l_{\mathrm{d}}$ est lare dune chaînette horizontale $p q$ de modules $m=\frac{\mathrm{T}_{0}}{\mathrm{II}}$, de portée $2 d$. Le rapport de similitude entre cette chấnette et celle de référence est : $\frac{m}{r}$; c'est aussi le rapport de similitude entre le segment incliné de la chânette dont fait partie

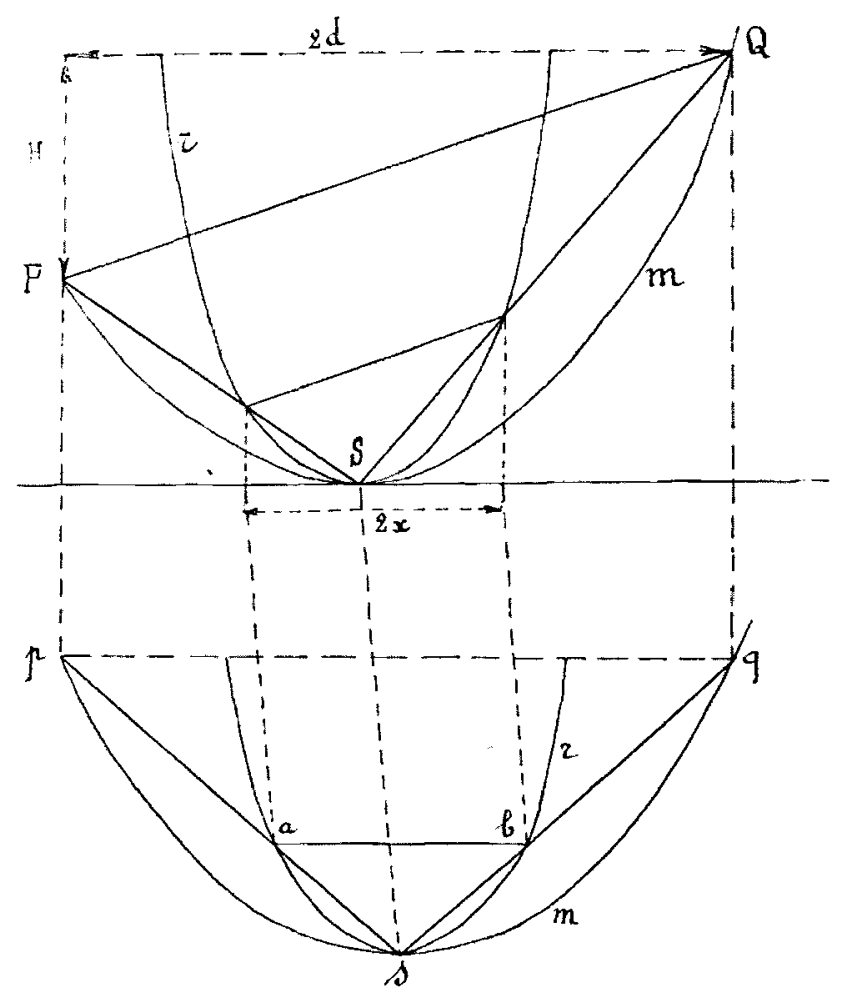

Fig. 6.

le câble et le segment semblable de la chaînette de référence; comme, d'autre part, ce dernier rapport est aussi $\frac{2 d}{2 x}$, on a :

$$
\frac{p q}{a b}=\frac{m}{r}=\frac{2 d}{2 x}
$$

comme $p q$ est égal à $2 d$, il en résulte :

$$
a b=2 x
$$

d'où la construction représentée fig. 6 . La longueur " $2 l_{\mathrm{d}}$ " est celle de l'arc $p s q$ de la chainette de module $m$ et de portée $2 d ; 2 l_{\mathbf{x}}$ est la longueur de l'arc $a s b$ de la chaînette de module $r$. On a :

$$
2 l_{\mathrm{d}}=2 l_{\mathrm{x}} \cdot \frac{2 d}{2 x}
$$

done :

$$
\mathrm{L}=\sqrt{\mathrm{H}^{2}+\left(2 l_{\mathrm{x}} \frac{2 d}{2 x}\right)^{2}}
$$

La tension moyenne du nouvel équilibre est (voir formule $9^{\text {bis }}$ ) :

$$
\mathrm{T}_{\mathrm{m}}=\frac{\mathrm{T}_{0}}{2 \mathrm{I}_{0}}\left[2 d+\mathrm{l}_{\mathrm{a}}\left(1+\frac{\mathrm{H}^{2}}{2\left(l_{\mathrm{x}} \frac{2 d}{2 x}\right)^{2}}\right)\right]
$$

La est la domie longueur d'un are de chaînetle horizontale de portée $2.2 d$ et de module $m$ : la porlée semblable de la châ̂netle de réference est $2.2 d, \frac{r}{m}$ c'est-à-dire $2.2 d . \frac{2 x}{2 d}$ c'est-àdire 2.2x. Done:

$$
\lg a=\frac{1}{2} \cdot 2 \cdot \ln x \frac{2 d}{2 x}=\lg \times \frac{2 d}{2 x}
$$

comme, d'antre part :

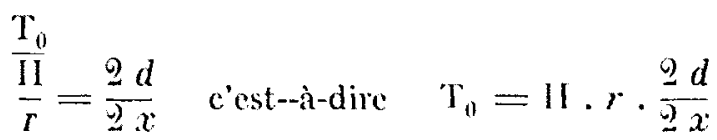

la formule précédente devient :

$$
\mathrm{T}_{\mathrm{m}}=\frac{\mathrm{II} \cdot r \cdot \frac{2 d}{2 x}}{2 \mathrm{I}_{0}}\left[2 d+l_{2 \mathrm{x}} \cdot \frac{2 d}{2 x}\left(1+\frac{1 H^{2}}{2\left(l_{\mathrm{x}} \frac{2 d}{2 x}\right)^{2}}\right)\right]
$$

I a formule (7) donne alors :

$$
\begin{gathered}
\sqrt{\mathrm{H}^{2}+\left(2 l_{\mathrm{x}} \frac{2 d}{2 x}\right)^{2}}=I_{0}\left[1+i \frac{\operatorname{II} \frac{2 d}{2 x}}{2 L_{0}}\right. \\
\left.\ldots\left[2 d+\log \frac{2 d}{2 x}\left(1+\frac{\mathrm{H}^{2}}{2\left(l_{x} \frac{2 d}{2 x}\right)^{2}}\right)\right]\right]
\end{gathered}
$$

Pour chaque valeur de $x$, les tables de la chaînetle de référence donnent $l_{\mathrm{x}}, l_{\mathrm{x}}$, d'où, pour le premier membre, une valeur $z$, el, pour le deuxième membre, une valeur $z$. Par approximations successives et par la méthode graphique habituelle en pareil cas, on détermine la valeur $x_{0}$ de $x$ pour laquelle $z=z$ '. L'équation (21) donne alors la tension cherchée:

$$
\mathrm{T}_{0}=I I \cdot r \cdot \frac{2 d}{2 x}
$$

Remarque. - Malgré la grande facilite quapporte aux calculs de (23) l'établissement préalable des Lables $l_{\mathrm{x}}, l_{\mathrm{x}}$, il cst incontestable que l'élégance el la rapidilé des deux méthodes résumées aux paragraphes précédents condamneraient celle-ci si on l'employait dans les cas ou l'une des deux autres suffit. Aussi est-elle à envisager seulement pour les très grandes portées car, alors, on ne doit pas reculer devant la nécessité des calculs.

Néanmoins, afin de pouvoir comparer ses résultats à ceux donnés par les deux premières, je l'appliquerai à un des exemples traités par M. Brachet.

Exemple :

$2 d=150 \mathrm{~m} . \quad \mathrm{H}=150 \mathrm{~m} . \quad \mathrm{T}_{\mathrm{i}}=8 \mathrm{~kg} \quad \pi=0,009 / 4$

$$
\text { II }=1,71 . \pi
$$


Je prends comme chaînette de référence $r=281,69$, pour la seule raison que j'ai antérieurement ( ${ }^{1}$ ) établi ces tables.

licmplaçant les lettres de l'exemple général ci-dessus par ces valeurs, on obtient :

par la formnle (17): $\quad 2 x_{\mathrm{i}}=24,8239$

Dans les tables de $\quad r=281,69$ on trouve :

pour $x=24 \quad \hat{\varepsilon}_{\mathbf{x}}=0,029$

pour $x=26 \quad \Sigma_{\mathrm{x}}=0,0369$

Au moyen de ces dilférences premières, on calcule par la formule de Newton :

$$
\varepsilon_{\mathbf{x}}^{\mathrm{i}}=0,0321
$$

done :

$$
l_{\mathrm{x}}^{\mathrm{i}}=24,8239+0,0321=24,855
$$

Par application de $\left(17_{\text {bis }}\right)$ :

$$
l_{\mathrm{d}}=24,855 \frac{\frac{8}{0,11094}}{281,69}=75,(194
$$

et par application de (18) :

$$
\mathrm{L}_{\mathrm{i}}=\sqrt{\overline{150^{2}}+\mathrm{L} \cdot(75,1094)^{2}}=212, \mathrm{~m} 265
$$

Dans les tables de $" r$ " on trouve ensuite : $\left({ }^{2}\right)$

$$
\begin{array}{ll}
\text { pour } 2 x=50 & \varepsilon_{\mathrm{x}}=0,2618 \\
\text { pour } 2 x=52 & \varepsilon_{\mathrm{x}}=0,2793
\end{array}
$$

qui permettent de déterminer :

donc :

$$
\tilde{z}^{i} \mathrm{x}=0,2569
$$

et par suite :

$$
l_{z x}=49,9048
$$

$$
l_{: x}=49,9048 \cdot \frac{\frac{8}{(1,009 / 4}}{281,69}
$$

Par application de (19) :

$$
\begin{aligned}
& \mathrm{T}_{\mathrm{m}}=\frac{8}{2 \cdot 212,265}\left[150+49,9048 \cdot \frac{8}{2,64788} .\right. \\
& \left.\ldots\left(1+\frac{\overline{150^{2}}}{2(75,094)^{2}}\right)\right]=11 \mathrm{k}, 32 \mathrm{R}
\end{aligned}
$$

(20) donne alors :

$$
\mathrm{L}_{0}=\frac{212,26 \mathrm{i}}{1+\left(17 \cdot 10^{-6} \cdot 11,328\right.}=212 \mathrm{~m}, 032
$$

L'équation (233) permet maintenant d'ècrire directement :

$$
\begin{gathered}
\sqrt{150^{2}+\left(2 l_{x} \frac{150}{2 x}\right)^{2}}=212,032 \\
\ldots\left[1+\lambda \cdot \frac{1,71.0,009 \cdot 4 \cdot 281,69 \cdot \frac{150}{2 x}}{2.212,(032}\left[150+\ln \times \frac{150}{2 x}\right.\right. \\
\left.\ldots\left(1+\frac{\overline{150}^{2}}{2\left(l_{x} \frac{150}{2 x}\right)^{2}}\right)\right]
\end{gathered}
$$

(1) Voir Houille Blanche de novembre-déccmbre 1925.

(2) Le calcul de $\varepsilon_{x}$ n'avait pas été fait par $2 x=48$. c'est-à-dire après calcul des coefficients et simplifications :

$$
\begin{gathered}
150 \sqrt{1+\left(\frac{l_{x}}{x}\right)^{2}}-212,032=2,47\left[\frac{1}{x}+\frac{l_{2 x}}{l_{x}^{2}}\right. \\
\left.\ldots\left(1+\frac{1}{2}\left(\frac{l_{x}}{x}\right)^{2}\right)\right]
\end{gathered}
$$

Appelons $z$ le premier membre et $z^{\prime}$ le second.

Pour $n=25$ les tables de $r$ donu $n t \quad\left\{\begin{array}{l}l_{\mathrm{x}}=25,0329 \\ l_{2 \mathrm{x}}=50,2628\end{array}\right.$ d'où :

$$
z=0,233 \quad z^{\prime}=0,396
$$

Pour $x=26$ les tables de $r$ donnent $\left\{\begin{array}{l}l_{\mathbf{x}}=26,0369 \\ l_{\mathbf{2}}=52,2958\end{array}\right.$ d'où :

$$
z=0,248 \quad z^{\prime}=0,3807
$$

Nous voyons que $z^{\prime}-z$ est passé seulement de 0,163 à 0,1327 . En admettant une certaine proportionnalité entre $\Delta\left(z^{\prime}-z\right)$ et $\Delta x$, on voit que $z$ sera égal à $z$ pour $x$ voisin de 30 .

J'essaye alors $x=35$

Les tables de $r$ donnent : $l_{\mathrm{x}}=35,090 \quad \lg _{\mathrm{x}}=70,7226$ d'où :

$$
z=0,374 \quad z^{\prime}=0,2837
$$

$z^{\prime}-z$ étant devenu négatif, je reviens en arrière :

$$
x=27,5 \text { donne } z=0,263 \quad z^{\prime}=0,36037
$$

Enfin $x=30$ donne : $z=0,299 \quad z^{\prime}=0,3304$.

Le graphique $z^{\prime}-z=f(x)$ construit à grande échelle et compensé au moyen des différences troisièmes de l'ordonnée, est une ligne presque droite, légèrement incurvée vers le haut, dont l'intersection avec l'axe des $x$ est :

$$
x_{0}=31,3
$$

La valeur des $\mathrm{T}_{0}$ se déduit de (21) :

$$
\mathrm{T}_{0}=1,71.0,0094.281,69 \cdot \frac{150}{2.31,3}=10 \mathrm{k}, 85
$$

La valeur de la tension moyenne se déduirait de (22) mais elle n'a pas grand intérêt numérique.

La tension au point de contact de la tangente parallèle à $\mathrm{PQ}$ est :

$$
\mathrm{T}=\frac{\mathrm{T}_{0}}{\cos \alpha}=\mathrm{T}_{0} V \overline{\overline{2}}=15^{\mathrm{k}, 3}
$$

Comparons maintenant ces résultats à ceux obtenus par application des deux premières méthodes exposées précédemment.

Celle du $\S^{-1}$ serait conduite ainsi :

Tension initiale du point de contact de la tangente parallèle à $\mathrm{P} Q$ :

$$
\frac{8}{\cos x}=11,9
$$

En entrant dans l'abaque Blondel avec :

$$
\text { portée }=\sqrt{\overline{150}^{2}+1 \overline{150}}=212,13 \text { et tension }=11,3
$$

on obtient un point sur l'horizontale 81 .

Pour en déduire les conditions d'équilibre en surcharge II. il faut $y$ entrer avec :

$$
\text { portée }=212,13+1,71=362,74
$$


ef prendre l'intersection avee l'horizonlale $\$ 1$ qui dome comme tension au point de contact avec la tangente:

$$
16 k, 5
$$

au lieu de $15 k, 30$ trourés précédemment par la méthode de similitude.

Quand à la méthode du $\$ 2$, elle a dome a M. Brachet $T_{0}=11^{k}$ au lieu de 10,85 obtenus ci-dessus par la methode de similitude.

Nota. - Il est à remarquer que l'exactitude de la methode de similitude, bien que pouvant théoriquement ètre absolue, est fortement attenuee, pour les portées de faible amplitude, comme c'est le cas ici, si on ne conserve pas, dans chaque opération, au moins quatre décimales exactes.

III.- LitilisatioN dU PROCEDÉ "PAR SIMILITLDE"( ${ }^{1}$ ) LORSQUE LA TENSION DU CABLE AU POINT LE PLUS HAUT EST TRES DIFFÉRENTE DE LA TENSION MOYENAE.

1. La question des très longues portées se pose habituellement ainsi en pays accidenté :

Choisir un métal, une section de càble et des points d'attache tels que :

La distance du sol au câble soit toujours inférieure à la limite imposée.

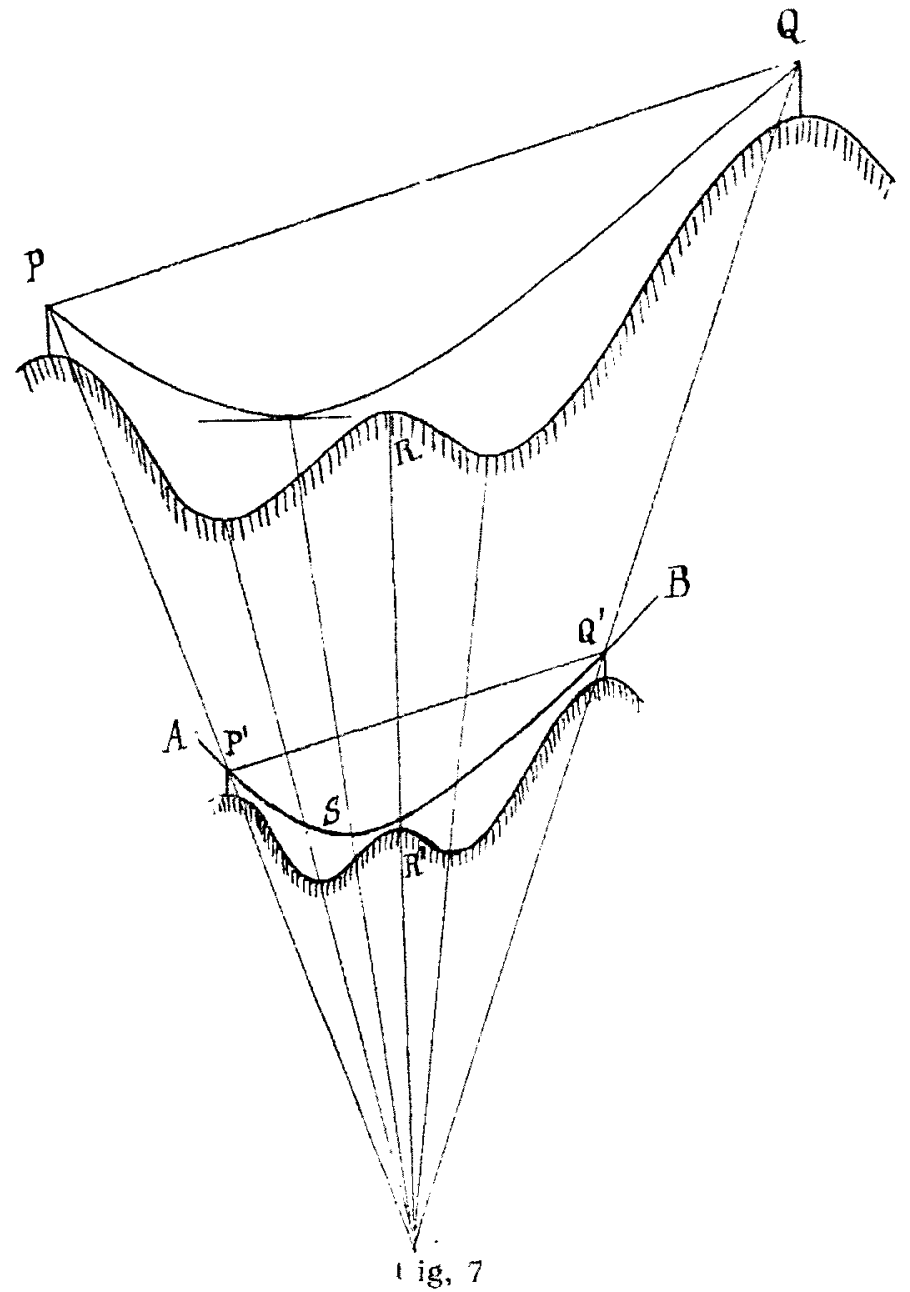

La composante verticale des forces au point d'attache du bas soit toujours dans le sens de la pesanteur.

(1) Ce procédé a, en pauticulier, permis a la "Société pour les Applications de l'Aluminium et des Alliages légers de Grenoble ", de vérifier avec exactitude ses calculs de portées de 1.200 et 1.400 mètres, projetées pour une ligne en aluminium-acier actuellement en construction dans la vallée de la Boume.
La tension an point le plus haul soit tonjours inféricure a celle résultant du taux de sécurité imposé.

On peut alors prendre le problème de deux facons différentes :

ler cas. - Ou bien déterminer d'abord graphiquement quelle est la chaînette la moins tendue (pas de surcharge et température maxima) la micux appropriec au terrain el chercher ensuile par le calcul pour un métal el une section donnés quelle sera la tension maxima d'un càble place ainsi, lorsque les conditions atmospheriçues so modifieraient.

$2^{e}$ cas. - Ou bien déterminer pour une portée donnéc la section du câble et son métal de manière à salisfaire aux conditions de surcharge les plus dures et chercher ensuite si le câble ainsi posé se maintient en tous ses points à une distance suffisante du terrain lorsque les conditions atmosphériques varient.

Dans le 1er cas, on dessinera d'abord la coupe du terrain et des points d'attache $\mathrm{P} Q$, ainsi que la chânette de référence A B. Les calquer ensuite sur deux feuilles transparentes $p q, a b$,

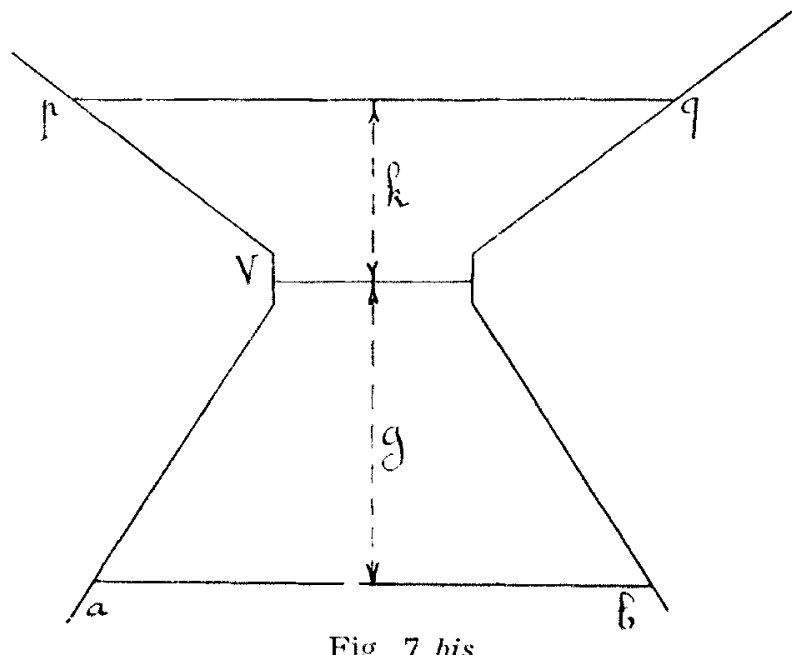

Fig. 7 bis

que l'on placera sur deux plaques de verre dépoli dans un appareil à agrandissement $V$; en éclairant $p q$ l'image de $P R Q$ se projettera sur ASB. Par déplacements relatifs de l'objectif o et par glissements des deux calques sur les plaques de leurs châssis, on cherchera la position pour laquelle l'image $P^{\prime} R^{\prime} Q^{\prime}$ de $P R Q$ vient se placer tangentiellement à A SB ; dans cette position, on immobilisera, par un léger collage, les deux calques sur leurs chảssis, puis on rapprochera $a b$ suffisamment pour que les points de P'R' $Q$ ' les plus voisins de ASB en soient à une distance suffisante (limite imposée, transposée à l'échelle de AB). Mesurer alors $g, k$ et calculer :

$$
F^{\prime}=\frac{g}{k} \times r \text { apport des échelles des deux dessins. }
$$

Placer ensuite sur A B la figure semblable au terrain ainsi réalisée; cela revient à matérialiser sur la feuille de dessin la figure 7. Il sufîit pour cela de promener parallèlement à l'inclinaison de $P Q$ une droite de longueur F.PQ dont une exlrémilé est appuyée sur la courbe $\triangle \mathrm{SB}$ jusqu'à ce que l'autre extrémité vienne aussi sur cette courbe. Il ne resle plus qu'à vérifier l'exactitude du résultat, en dessinant entre les deux poinls de $\triangle \mathrm{SB}$ ainsi obtenus la coupe du terrain à l'échelle $F$, ce qui permet de s'assurer que l'écartement limite n'est alleint en aucun point particulier tel que $\mathrm{R}$ '.

Ia chânelte limite convenant au termain a comme moduk, en appelant $B$ celui de ba chañete re référence du dessin :

$$
m=-\mathrm{R}
$$


Les formules du chapitre I permettant de déterminer les divers éléments de celte chaînelte, on peut, en particulier, vérifier par le calcul qu'aucun point du terrain ne s'en approche à un intervalle trop faible.

Se donner ensuite un métal et une seclion du câble (c'est-àdire $\pi$ ), ainsi que la température maximum $\Theta$.

Puis déterminer, au moyen des formules déduites par le procédé de similitude, comme on l'a fait précédemment, de celles du chapitre I, la tension maxima du câble ainsi calculé, et dans le cas le plus défavorable. Si cette tension est supérieure à celle résultant de la limite de sécurité imposée, modifier soit l'emplacement du point d'altache soit le métal, soit la section.

Dans le $2^{\mathrm{e}}$ cas. - Pour un diamètre donné $\mathrm{D}$, on connaît la surcharge maxima II produite à une certaine température $\theta$ par la résultante du poids et du vent ou du givre; d'autre part pour le dianètre D el pour chaque métal, on connaît la tension maxima T admissible. Il s'agit maintenant de savoir si un câble dı métal choisi, de diamètre $\mathrm{D}$, tendu entre $\mathrm{P}$ et $\mathrm{Q}$ de manière à ce que la tension en $\mathrm{P}$ soil $\mathrm{T}$ pour une charge II réalisée à la tempéralure 1, salisfera au profil du terrain pour toutes les conditions atmosphériques el si la tension maxima $\mathrm{T}$ ne sera jamais dépassée.

En d'autres tormes la chaînette iniliale étant déterminée par :

$$
\mathrm{Y}_{\mathrm{i}}=\frac{\mathrm{T}}{\mathrm{II}}
$$

au point $Q$, ainsi que par la pente, la portée $P Q$, le coefficient de dilatation $x$, le coefficient d'allongement $\pm \lambda$, il faut voir ce qu'elle devient quand la température passe de $\theta$ à $\Theta$ et la charge de $I I$ à $\pi$. Cela fait, il ne restera plus qu'à la poser sur la coupe du terrain par le procédé graphique exposé au premier (as, avec, cependant, cette simplification, que, le module de la chaînelte finale ayant été calculé, et le rapport de similitude graphique $\mathrm{F}$ étant par conséquent connu, on pourra placer directement $P^{\prime} Q^{\prime}$ sur le dessin sans avoir à se servir de l'appareil à agrandissement.

La question est donc entièrement résolue théoriquement par les formules déduites, au moyen du principe de similitude, comme on l'a fait précédemment, de celles des paragraphes 6 et 7 du chapitre I. Mais j'ai annoncé dans ce chapitre la possibilité de simplifier les calculs par des constructions graphiques. Un exemple numérique va me donner l'occasion d'exposer ces constructions.

Bien que cet exemple eût dû être choisi parmi les portées exceptionnelles, j'ai pris à dessein une portée moyenne à pente ordinaire afin de rester dans des limites permettant des comparaisons avec les résultats que donneraient les méthodes utilisant les abaques de M. Blondel.

\section{Domnées initiales :}

Câbles aluminium de 37 brins de $\frac{382}{100}$

Tension maxima admise par $\mathrm{mm}^{2}: 11 \mathrm{~kg}$

Projection horizontale de la portée : $2 d=170 \mathrm{~m}$.

Différence de niveau des points d'altache : $H=14 \mathrm{~m} .45$

Hypothèse qui semble la plus défavorable $\left(^{(}\right)$: vent de $72 \mathrm{~kg}$. a $10^{\circ}$.

Poids unitaire apparent correspondant à cette hypothèse, par $\mathrm{mm}^{2}$ de section utile : II $=0,00385 \times 1,61$

(1) Voir plus loin remarque faite à ce sujet, à propos de la longueur initiale.
Dessiner d'abord très exactement à une échelle convenable une chaînette auxiliaire calculée au moyen des tables de la chấnette de référence $r$; cette chaînette auxiliaire de module $\mathrm{R}$ devra être choisie de manière à être utilisable pour tous les câbles et toutes les portées d'une même catégorie afin de n'avoir pas à recommencer pour chaque projet ce dessin préliminaire. Dans le but de tirer partie de la remarque faite au chapitre I formule 7 ter, il sera avantageux de dessiner aussi la chaînette de module $\frac{\mathrm{R}}{\mathrm{Q}}$.

Si on a à étudier des portées peu inclinées, il sera préférable de prendre l'échelle des $y$ plus grande que celle des $x$. Après quelques tâtonnements, on arrive à se formuler des règles pratiques qu'il serait trop long d'exposer ici et que chacun peut d'ailleurs déterminer à sa manière, desquelles on déduit certains repères permettant de faire un choix judicieux de $R$ et des échelles.

Dans le cas des câbles aluminium acier à 37 brins et des portées du genre de celle choisie ici, j'ai pu utiliser une chaînette auxiliaire de module quadruple de la chaînette de référence utilisée dans l'exemple traité an chapitre précédent.

$$
\mathrm{R}=4 . r=4.281,69 \text {. }
$$

Les échelles de l'épure sont $\left\{\begin{array}{l}\text { pour } x: 1 \mathrm{~m} / \mathrm{m} \text { par mètre. } \\ \text { pour } y: 10 \mathrm{~m} / \mathrm{m} \text { par mètre. }\end{array}\right.$

\section{a) Construction graphique initiale}

Il s'agit de placer sur cette courbe une corde $\mathrm{P}^{\prime} \mathrm{Q}^{\prime}$ de pente

telle que (fig. 8) :

$$
\frac{14,45}{170} \cdot \frac{10}{1}
$$

$$
\frac{y+10 . \mathrm{R}}{\mathrm{Y}_{\mathrm{i}}}=\frac{\mathrm{P}^{\prime} \mathrm{Q}^{\prime}}{\mathrm{PQ}}
$$

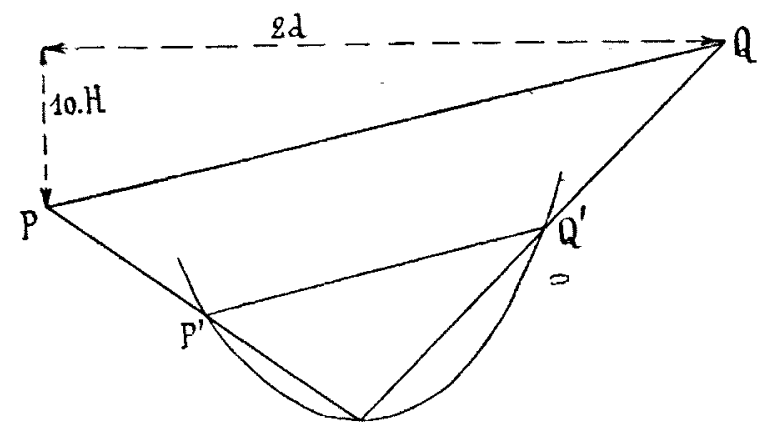

Fig. 8

étant entendu que :

$$
\mathrm{Y}_{\mathrm{i}}=10 \cdot \frac{11 \mathrm{~kg}}{0,00385 \cdot 1,61}
$$

Pour faciliter l'explication de la construction adoptée (fig. 9), je suppose que l'inclinaison donnée est AB. Soit $a b$, sur la chaînette auxiliaire, la corde parallèle à $\mathrm{A} B$, à déterminer de telle façon que :

$$
\frac{a b}{\mathrm{AB}}=\frac{y+10 \cdot \mathrm{R}}{\mathrm{Y}_{\mathrm{i}}}
$$

Soit $\mathrm{AK}=\mathrm{Y}_{\mathrm{i}}$ et $a k=y+10 \mathrm{R}$. Les triangles $b d a^{*}$ $\mathrm{BD} A$ sont semblables, de même ade, ADE. On a donc.:

$$
\frac{\mathrm{A} \mathrm{D}}{\mathrm{ED}}=\frac{a d}{d e}=\frac{f g}{g e}
$$


D'où :

$$
f g=g e \cdot \frac{\mathrm{Al}}{\mathrm{ED}}=10 \cdot \mathrm{R} \cdot \frac{\mathrm{AD}}{\mathrm{I}_{\mathrm{i}}}
$$

Dans le cas actuel:

$\mathrm{AD}=170 \mathrm{~m} / \mathrm{m} \quad \mathrm{Y}_{\mathrm{i}}=\frac{11}{0,(1035.1,61}, 10 \mathrm{~m} / \mathrm{m} \quad \mathrm{R}=4.281,69$

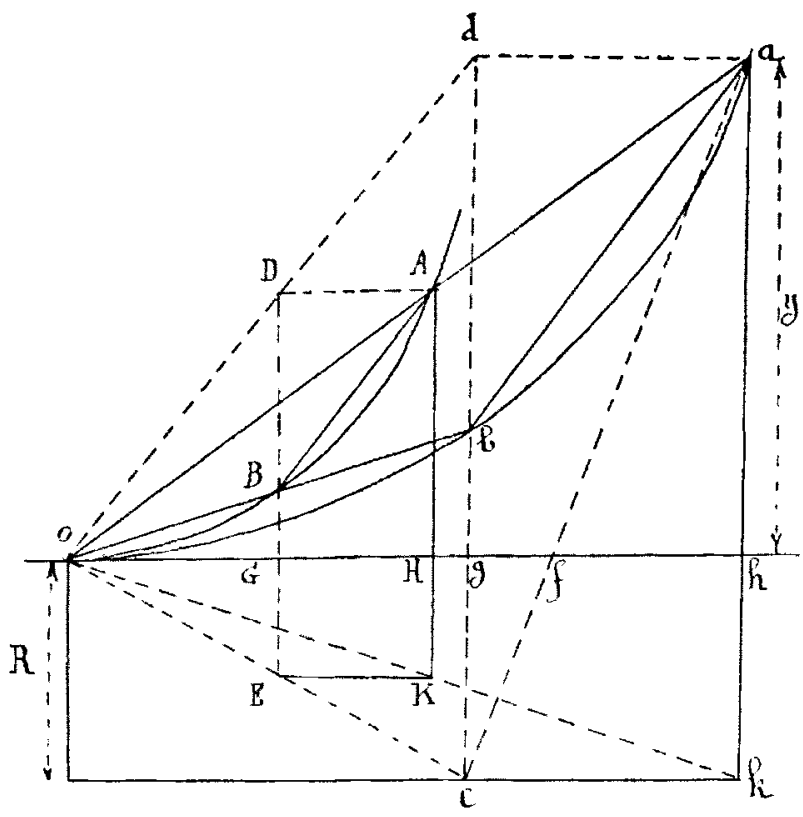

Fig. 9

et on trouve :

$$
f g=107 \mathrm{~m} / \mathrm{m} 9=26,975 \times 4
$$

D'autre part, puisque $\mathrm{KE}=170 \mathrm{~m} / \mathrm{m}$ et $\mathrm{AK}=\mathrm{Y}_{\mathrm{i}}$, la direction $\mathrm{AE}$, c'est-à-dire la direction de $a /$, peut ètre construite graphiquement.

Pour déterminer la position de $a b$ sur la chaînette auxiliaire O R on opèrera alors ainsi (fig. 10) :

Prendre un point quelconque $a^{\prime}$ sur OR, mener la droite $a^{\prime} f^{\prime}$ de direction connue (celle de $a f$ ); porter f' $q^{\prime}$ égal à $107 \mathrm{~m} / \mathrm{m} 9$; mener la verticale $g^{\prime} b^{\prime}$ et, par $a^{\prime}$, une droite parallèle à $\mathrm{AB}$; elle coupe $g^{\prime} b^{\prime}$ en un point $b_{1}$.

Recommencer pour un autre point $a^{\prime}$ jusqu'à ce que $b^{\prime} b_{1}$, soit nul.

Cette construction donne toute satisfaction quand $g^{\prime}$ est voisin de $o$, car alors l'intersection $b$ ' est très franche.

S'il n'en est pas ainsi, on peut, de la figure 9, déduire d'autres constructions géométriques permettant de vérifier les résultats de la première. Pour ne pas allonger outre mesure cet article, je m'en tiendrai à celle-ci.

On trouve, par cette construction :

$$
\begin{array}{ll}
x_{1}^{\prime}=40 \mathrm{~m} / \mathrm{m} & y_{1}^{\prime}=6 \mathrm{~m} / \mathrm{m} 8 \\
x_{2}^{\prime}=151 \mathrm{~m} / \mathrm{m} & y_{2}^{\prime}=102 \mathrm{~m} / \mathrm{m}
\end{array}
$$

Ces coordonnées rapportées à la chaînette de référence $r=\frac{\mathrm{R}}{4}$, et exprimées en mètres, deviennent :

$$
\begin{array}{ll}
x_{1}=10 \mathrm{~m} . & y{ }_{1}=0 \mathrm{~m} 170 . \\
x^{\prime \prime}{ }_{2}=37 \mathrm{~m} 8.5 & y^{\prime \prime}{ }_{2}=2 \mathrm{~m} 55 .
\end{array}
$$

Mais les calculs qui voul suive exigeant une très grande précision, ces coordonnées graphiques ne peuvent être adoptées saus corrections,
En calculant, au moyen des tables de $r$, l'y correspondant a $x_{1}=10$, on trouve : $y_{1}=0 \mathrm{~m} \mathrm{177}$. D'autre part (fig. 9), les triangles semblables o $\mathrm{B} \mathrm{E}$, $o b c$, donnent :

$$
\frac{b e}{\mathrm{BE}}=\frac{o b}{O \mathrm{~B}}
$$

c'est-à-dire :

$$
\frac{y_{1}+r}{Y_{1}-1 \cdot 4,45}=\frac{o b}{o B}
$$

et comme :

$$
\frac{o b}{o \mathrm{~B}}=\frac{o a}{o \mathrm{~A}}=\frac{a d}{\mathrm{~A} \mathrm{D}}=\frac{x_{2}-x_{1}}{170}
$$

on a donc :

$$
\frac{y_{1}+r}{Y_{i}-14,45}=\frac{x_{2}^{\prime \prime}-x_{1}}{170}
$$

d'où on tire :

$$
x_{2}^{\prime \prime}=x_{1}+170 \cdot \frac{y_{1}+r}{\mathrm{Y}_{\mathrm{i}}-1 /, 15}
$$

qui domne:

$$
\begin{aligned}
x_{2}^{\prime \prime} & =x_{1}+170 \cdot \frac{(0,177+281,69)(1,00385 \cdot 1,61}{11-1 / 4,45 \cdot 0,00385 \cdot 1,61} \\
& =10+27,224=37,224
\end{aligned}
$$

au lieu de 37,85 trouvé graphiquement.

Mais si on détermine $x_{2}$ par l'intersection de la chainette " $r$ " avec une droite parallèle à A B passant par le point $x_{1} y_{1}$ c'est-àdire par l'équation :

$$
\frac{14,45}{170}=\frac{y_{2}-y_{1}}{x_{2}-x_{1}}=\frac{r\left(\cosh \frac{x_{2}}{r}-\cosh \frac{x_{1}}{r}\right)}{x_{2}-x_{1}}
$$

qu'on résoul soil en employant une lable hyperbolique soil

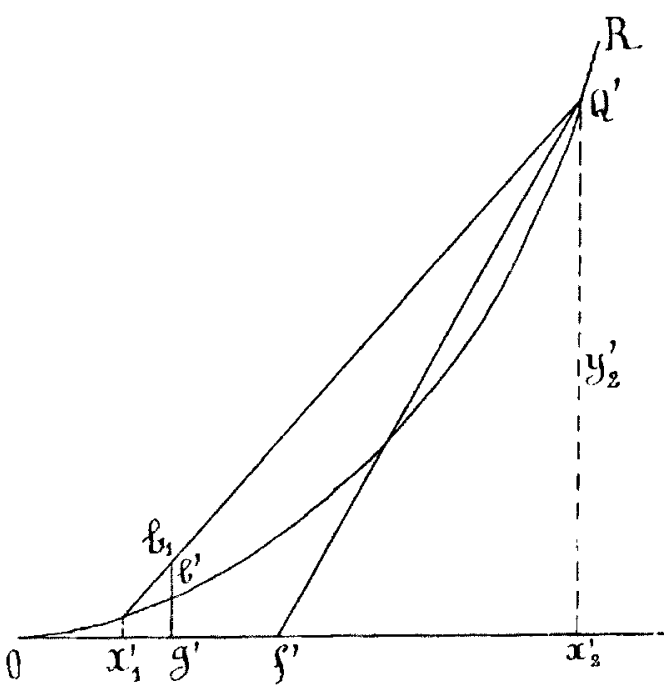

Fig. 10

au moyen des tables de " $r$ ", par approximations successives, on trouve :

$$
x_{2}=37,822 \text { d'où } y_{2}=2,5429
$$

b) Faut-il prendre 37,224 on 37,822 ?

Pour le décider, cherchons l'explication de cetle divergene.

Le point $b$ devrait ètre l'intervention exacte de ols avec la chaînette $o \mathrm{R}$; le point oblenu graphiquement en diflère légère- 
ment de sorte que l'abscisse lue $x_{1}=40$ correspond à un poinl $b_{1}$ de $o \mathrm{R}$ dont l'ordonnée est $y_{1}^{\prime}=0,177 \times 4$. Les égalités $(24)$ el (25) élanl basées sur le parallélisme de la droite B A et de celle à oblenir ainsi que sur la proportionnalité de leurs longueurs, amèneront à un point $a_{1}$ tel que $a_{1} a b b_{1}$ soit un parallélogrammə de sorte que ce point $a_{1}$ d'abscisse $37,224 \times 4$ n'est nullement sur la chaînelte $o R$ et que, par suite, il ne peut servir à déterminer le rapport de similitude cherché. - Au contraire l'équation (26) exprime que le point $4 x_{2}, 4 y_{2}$ est à l'intersection de la chaînelte $o \mathrm{R}$ et de la ligne droite $b_{1} a_{1}$; ce point est $a_{2}$. Il n'y a donc aucun doute, il faut adopler $x_{2}=37,812$ et non pas 37,224 .

Mais il est bon de se rendre compte des conséquences de ce choix. Ce point $a_{2}$ devra correspondre à un point $A_{2}$ tel que $A_{2} B_{1}$

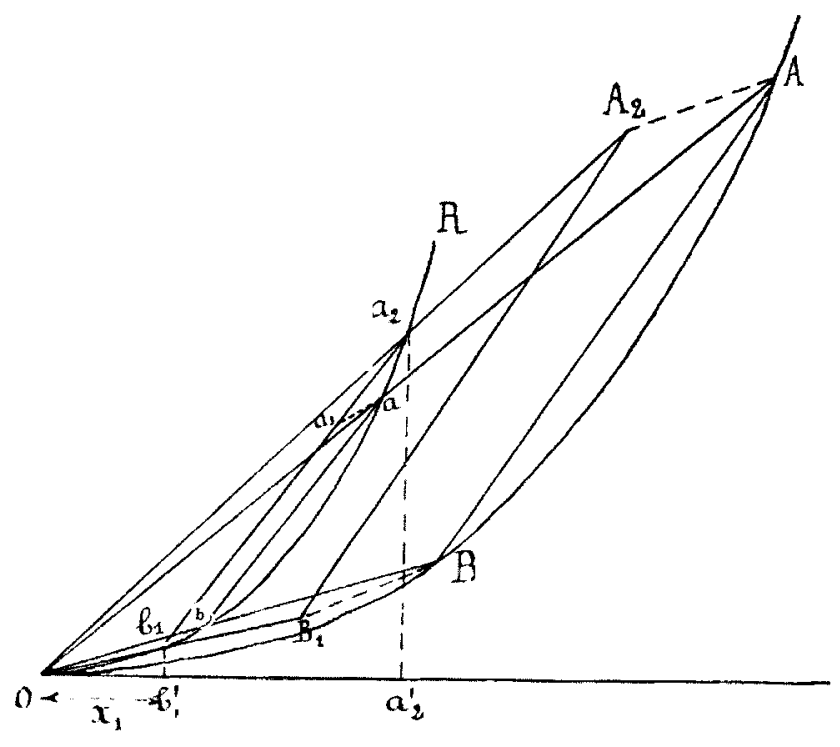

Fig. 11

ait la longueur et l'inclinaison $\mathrm{AB}$ c'est-à-dire que $\mathrm{A}_{2} \mathrm{ABB}_{1}$ est un parallélogramme. La chaînetle cherchée étant semblable à la chaînette " $r$ » et le rapport de similitude étant

$$
\frac{\frac{a_{2} b_{1}}{4}}{\frac{A_{2} B_{1}}{4}} \text { c'est-à-dire } \frac{\frac{a_{2}^{\prime} b_{1}^{\prime}}{170}}{170} \text { c'est-à-dire } \frac{\frac{(37,829-10)}{4}}{170} \text {, }
$$

son module $m$ satisfait à la relation :

$$
\frac{m}{r}=\frac{170}{37,822-10}
$$

c'est-à-dire :

$$
m=281,69 \cdot \frac{170}{27,822}=1721 \mathrm{~m}, 20
$$

La tension horizontale de cette chaînette est :

$$
\mathrm{T}_{\mathrm{o}}^{\mathrm{i}}=1721,2 \times \mathrm{II}=10 \mathrm{k} .668
$$

Quel est l'X de celte chainette au point $x_{2} y_{2}$, c'est-à-dire quelle est la tension maxima initiale?

Il sìlisfail à :

$$
\frac{\mathrm{Y}_{\mathrm{i}}}{y_{\mathrm{Q}}+r}=\frac{m}{r}=\frac{170}{27,822}
$$

Donc :

$$
\mathrm{T}_{\mathrm{i}}=11 \cdot\left(y_{2}+r\right) \frac{170}{27,829}=19 \mathrm{k}, 759
$$

L'erreur graphique initiale équivaut donc à une légère modification du taux de sécurité imposé; cette erreur peut d'ailleurs être d'un sens quelconque.

$\mathrm{Si}$ on voulait tomber exactement sur $11 \mathrm{k}$, il faudrait augmenter $m$, c'est-à-dire, puisque

$$
\frac{m}{r}=\frac{170}{x_{2}-x_{1}}
$$

diminuer $x_{2}-x_{1}$; comme $a b$ resterait parallèle à la même direction, $x_{2}$ et $y_{2}$ devraient ètre pris plus petits. On essayerait, par exemple, $x_{2}=37,820$ : pour cela, on calculerait l' $y_{2}$ correspondant, puis $x_{1} y_{1}$ intersection de la chaînette de référence $r$ avec une droite passant par $x_{2} y_{2}$ et parallèle à $\mathrm{A} B ; \frac{170}{x_{2}-x_{1}}$ donnerait un nouveau rapport de similitude duquel on déduirait, comme ci-dessus, $Y_{\mathbf{i}}$ puis là tension. Par approximations successives on pourrait ainsi trouver un point a donnant exactement $\mathrm{T}_{\mathrm{i}}=11$.

Mais il est bien évident que celte recherche minutieuse est généralement inutile, car de deux choses l'une : ou bien on dispose d'un métal déterminé et alors il vaut mieux adopter un taux de sécurité légèrement plus fort que celui imposé ce qui s'obtient en modifiant dans le sens voulu $x_{2} y_{2}$ résultant de la construction graphique, ou bien le fournisseur du métal dispose de toute une gamme de métaux et alors il y a avantage pour le constructeur de la ligne à adopter le $\mathrm{T}_{\mathrm{i}}$ le plus fort; dans ce cas, une différence de l'ordre de l'erreur résultant de la construction graphique n'aura aucune importance car $T_{i}=11 \mathrm{~kg}$ aura été choisie un peu au hasard et légèrement au-dessous du maximum réalisable par le fournisseur.

J'adopte donc : $\mathrm{T}_{\mathrm{i}}=10 \mathrm{~kg} .759$

vu la formule (18) :

$$
L_{i}=\sqrt{14,45^{2}+\left(2 l^{i} \times \frac{170}{27,820)}\right)^{2}}
$$

Comme vérification, appliquons la formule (17) :

$$
2 x_{\mathrm{i}}=170 \cdot \frac{27,822}{170}=27,822
$$

on retrouve donc bien pour $2 x_{\mathrm{i}}$ la valeur de $x_{2}-x_{1}$, comme $\mathrm{i}^{l}$ fallait s'y attendre.

Done :

$$
x_{\mathrm{i}}=13,911
$$

On pourrait déduire $l_{\mathrm{x}}^{\mathrm{i}}$ des tables de $r$ " par interpolation mais l'erreur commise ainsi serait multipliée par $2 \cdot \frac{170}{27,8}$ c'està-dire environ par 12 ; il est donc préférable de calculer $l_{\mathrm{x}}$ par la formule (2) on trouve :

$$
l_{\mathrm{x}}^{\mathrm{i}}=13,9167
$$

donc :

$$
L_{i}=\sqrt{208,8025+\left(2 \frac{170}{27,820} 1.3,9167\right)^{2}}=170,82
$$

La tension moyenne pent ètre évaluée graphiquement an moyen de la formule (7 ter) :

$$
\mathrm{T}_{\mathrm{m}}=\frac{\mathrm{T}_{0}{ }^{1}}{2 \mathrm{~L}_{\mathrm{i}}}\left[170+\frac{1}{2}\left(\mathrm{~L}_{\mathrm{x}_{2}}-\mathrm{L}_{\mathrm{x}_{1}}\right)\right]
$$


da ge parenthèse est la longueur du segment de la chantelte le module $\frac{m}{2}$ compris entre les verlicales $\mathrm{X}_{1}$ et $\mathrm{X}_{2}$ limitant le segment $A B$ de la chañette de module $m$. Si on considère les verticales $x_{1} x_{\mathbf{2}}$ de la chainette référence $r$ el la longueur $l_{\mathbf{x}_{\mathbf{z}}}-l_{\mathbf{x}_{\mathbf{t}}}$ du segment de la chainette $\frac{r}{2}$ compris entre ces verticales, on voit que ce segment est semblable au premier of que le rapport de similitude est :

$$
\frac{\frac{m}{2}}{\frac{r}{2}} \quad \text { c'est-at-dire } \frac{m}{2}
$$

donc :

$$
\mathrm{T}_{\mathrm{m}}^{\mathrm{i}}=\frac{\mathrm{T}_{0}{ }^{\mathrm{i}}}{2 \mathrm{~L}_{\mathrm{i}}}\left[170+\frac{1}{2} \cdot \frac{m}{r}\left(l_{\mathrm{x}_{\mathrm{t}}}-l_{\mathrm{x}_{\mathrm{s}}}\right)\right]
$$

ou bien, (fig. 12) :

$$
\mathrm{T}_{\mathrm{m}}^{\mathrm{i}}=\frac{\mathrm{T}_{0}{ }^{\mathrm{i}}}{2 \mathrm{~L}_{\mathrm{i}}}\left[170+\frac{1}{2} \frac{m}{r} \sqrt{b^{\prime} \mathrm{g}^{2}+\left[2 \frac{r}{2} \cdot \sinh \left(\frac{x_{\mathrm{i}}}{r}\right)\right]^{2}}\right]
$$

c'est-à-dire :

$$
\mathrm{T}_{\mathrm{m}}^{\mathrm{i}}=\frac{\mathrm{T}_{0}^{\mathrm{i}}}{2 \mathrm{~L}_{\mathrm{i}}}\left[170+\frac{1}{2} \frac{m}{r} \sqrt{\bar{b}^{2}+\left[2 \sinh \left(\frac{2 x_{\mathrm{i}}}{r}\right)\right]^{2}}\right]
$$

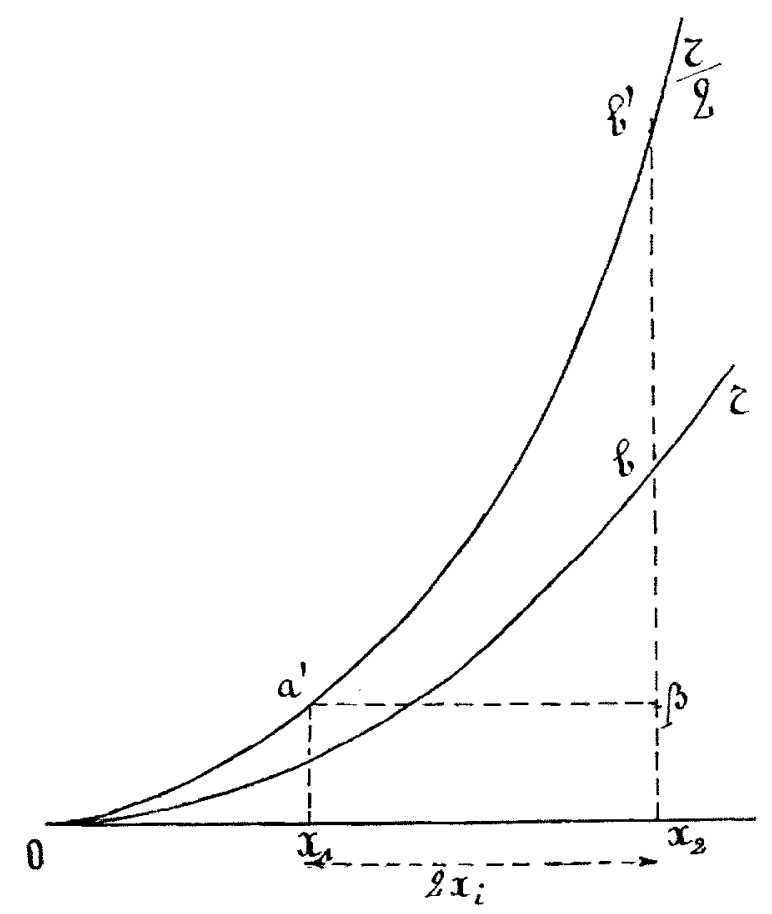

Fig. 12

ou

$$
\mathrm{T}_{\mathrm{m}}^{\mathrm{i}}=\frac{\mathrm{T}_{0}{ }^{\mathrm{i}}}{2 \mathrm{~L}_{\mathrm{i}}}\left[170+\frac{m}{2 r} V \overline{{\overline{b^{\prime} \beta^{2}}}^{2}+\left(l_{2} \mathrm{x}_{\mathrm{i}}\right)^{2}}\right]
$$

Sur l'épure des deux chaînettes auxiliaires $\mathrm{R}$ et $\frac{\mathrm{R}}{\mathrm{Q}}$ on peut mesurer la longueur $b^{\prime} \beta$; par suile de l'échelle des ordonnées choisie ici, il faudra diviser par 40 la valcur trouvée avant de la porter dans la formule ci-dessus. -- Quand à $l_{2} x$, elle est donnée par les lables de « $r$. On obtient ainsi :

$$
T_{m}{ }_{m}=\frac{10,688}{2.170,682}\left[170+\frac{170}{55,644} 56,25\right]
$$

Comme on l'a fait précédemment, il est prudent de connaître l'ordre de grandeur de l'erreur commise en opérant ainsi. A cet elfet, calculons $\mathrm{T}_{\mathrm{m}}^{\mathrm{i}}$ algébriquement par la formule 7 bis.

$$
\mathrm{T}_{\mathrm{m}}^{\mathrm{i}}=\frac{\mathrm{T}_{0}{ }^{\mathrm{i}}}{2 \mathrm{~L}_{\mathrm{i}}}\left[170+\frac{1}{2}\left(\mathrm{~L}_{2} \mathrm{x}_{\mathrm{s}}-\mathrm{L}_{\mathrm{g}} \mathrm{x}_{1}\right)\right]
$$

La $2^{e}$ parenthèse est la longueur du segment de la chainette de module " $m$ " compris entre deux verticales siluées à des distances de o doubles de celles passant par A et B. Ce segment est semblable à celui de la chânetle de référence compris entre les verticales $2 x_{1}$ et $2 x_{2}$; donc :

$$
\operatorname{Lg}_{x_{2}}-\operatorname{Le}_{x_{1}}=\frac{m}{r} \cdot\left(l_{2 x_{2}}-l_{2 x_{1}}\right)
$$

Egalite dans laquelle:

$$
2 x_{2}=75,644 \quad 2 x_{1}=20
$$

Les lables de i $r$ " clomment:

Done :

$$
l_{x_{2}}=76,+53 \quad l_{x_{1}}=20,0167
$$

$$
T_{m}=\frac{10,668}{2.170,682}\left[170+\frac{170}{55,61,50,516}\right]
$$

La difference entre celle valeur cxacle de $T^{i}{ }_{n}$ of ha valcur approchée précédente est :

$$
\frac{10,668}{2.170,682} \cdot \frac{170}{55,641}(56,516-56,2.7)
$$

c'est-à-dire environ 0 k. 032 soil $0,3 \%$. Comme $\mathrm{T}^{\mathrm{i}} \mathrm{m}$ sera multipliée ultérieurement par $\lambda$, la premiere manière semble être d'une exactitude suffisante pour les portées moyennes à forte tension.

J'adopte le $T_{m}^{i}$ calculé entièrement algćbriquement : $10 \mathrm{k} 7056$ La longueur du câble sans tension à $10^{\circ}$ résulte de l'équation (20):

$$
L_{0}^{i}=\frac{170,682}{1+\lambda .10,7056}
$$

avec $\lambda=1,15 \cdot 10^{-4}$; expression qui dome $L_{0}^{i}=170,472$ $a 0^{\circ}$, la longueur sans tension sera:

$$
\mathrm{L}_{0}{ }^{\circ}=\frac{170,472}{1+10 \cdot \alpha} \text { avec } \alpha=17,25.10-i
$$

Remarque. - 11 faudrait maintenant refaire des calculs analogues pour chacune des conditions initiales qui peuvent ètre les plus défavorables, et adopter pour $\mathrm{L}_{\mathbf{0}}{ }^{\circ}$ la plus grande des longueurs ainsi calculées.

Pour ne pas allonger outre mesure, j'admets que l'hypothese injliale choisie ici est la plus défavorable.

d) Il s'agit maintenant de voir si le câble ainsi calculé conviendra au profil du terrain quand le vent sera nul et la lcmpéralure $40^{\circ}$.

La longueur au repos à $40^{\circ}$ est :

$$
\mathrm{L}_{0}=170,172 \frac{1+10 \cdot \alpha}{1+10 \cdot \alpha}=170,567
$$

Sous la charge $\pi=0,00385$, celle longueur devient en appelant $2 x$ la projection du segment semblable de la chaînelle " $r$ " et 
$2 l_{\mathrm{x}}$ la longueur de la portée horizontale $2 x$ dans la chaînelte " $\Gamma$ " (formule 18') :

$$
\mathrm{L}=\sqrt{\overline{14,15^{2}}+\left(2 l_{\mathrm{x}} \frac{170}{2 x}\right)^{2}}
$$

la tension horizontale est devenue maintenant $\mathrm{T}_{0}$, et on a :

$$
\mathrm{T}_{0}=\pi \cdot r \cdot \frac{170}{2 x}=1,0845 \cdot \frac{170}{2 x}=\frac{184,36 i}{2 x}
$$

la tension moyenne esi devenue (formule 9 el 22) :

$$
\begin{aligned}
& \mathrm{T}_{\mathrm{m}}=\frac{\frac{184,363}{2 x}}{2 \sqrt{14,45^{2}+\left(2 l_{\mathrm{x}} \frac{1711}{2 x}\right)^{2}}} \\
& \cdots\left[170+\ln x \frac{170}{2 x}\left[1+\frac{\overline{1 / 4,15^{2}}}{2\left(l_{x} \frac{170}{2 x}\right)^{2}}\right]\right]
\end{aligned}
$$

et, comme, formule (7) :

il vienl :

$$
L=L_{0}\left(1+\lambda T_{n}\right)
$$

$$
\begin{aligned}
& \sqrt{\overline{14,45^{2}}+\left(2 l_{x} \frac{170}{2 x}\right)^{2}}=170,567 \\
& \ldots\left[1+115.10-i \cdot \frac{184,365}{4 x \sqrt{14,45^{2}+\left(2 l_{x} \frac{170}{2 x}\right)^{2}}}\right. \\
& \ldots\left[170+l_{2 \times} \frac{170}{2 x}\left(1+\frac{\sqrt{14,45^{2}}}{2\left(l_{x} \frac{170}{2 x}\right)^{2}}\right)\right]
\end{aligned}
$$

c'est-à-dire :

$x\left[835,25+115600\left(\frac{l_{\mathrm{x}}}{x}\right)^{2}-682,268 \sqrt{208,8+28900\left(\frac{l_{\mathrm{x}}}{x}\right)^{2}}\right.$

$$
\ldots=614,78+\frac{l_{x}}{2 x}\left[614,7 x+8,88\left(\frac{x}{l_{x}}\right)^{2}\right]
$$

Comme $\mathrm{T}_{0}$ est certainement inférieur à $\mathrm{T}_{0}^{\mathbf{i}}$, c'est-à-dire à 10,66 el comme

$$
2 x=\frac{184,365}{\mathrm{~T}^{0}}
$$

il en résulte : $x>9$.

Mais on peut, de plus, at moyen de cette expression de $x$, se faire une idée de sa valeur approximative.

$$
\begin{aligned}
& \text { Pour } x=40 \text { on aurail } \mathrm{T}_{0}=2,3 \\
& \text { - } x=30 \quad-\quad \mathrm{T}_{0}=3,4 \\
& \text { - } x=20 \quad-\quad \mathrm{T}_{0}=4,6 \\
& -x=10 \quad-\quad \mathrm{T}_{\mathrm{o}}=9,2
\end{aligned}
$$

Par analogie avec les cas usuels il est probable que To est compris entre $3 \mathrm{~kg}$. et $5 \mathrm{~kg}$.

J'essaye donc $x=30$.

Dans les tables de la chainette " $r$ " on trouve : d'où

$$
l_{\mathrm{x}}=30,0567 \quad \mathrm{Lq}_{\mathrm{x}}=60,454
$$

$$
\left(\frac{l_{x}}{x}\right)^{2}=1,0038 \quad\left(\frac{x}{l_{x}}\right)^{2}=11,9968 \quad \frac{l_{x}}{2 x}=1,00757
$$

Les valeurs correspondantes des deux membres de l'équation finale ci-dessus sont :

$$
30[835+1160: 37-116627] \text { et } 614,78+628,35
$$

La différence entre le $1^{\text {er }}$ et le $2^{\mathrm{e}}$ nombre est : 6125.

Le $2^{\mathrm{e}}$ membre varie lrès peu, $\frac{l_{2 x}}{2 x}$ et $\left(\frac{x}{l_{x}}\right)^{2}$ restant voisins de 1 ; dans le 1 er membre $\left(\frac{l_{x}}{x}\right)^{2}$ varie plus vite que

$$
\sqrt{208+\left(\frac{l_{x}}{x}\right)^{2} \cdot 28,900}
$$

d'ailleurs le premier est multiplié patr un facteur fixe plus fort que le second. Comme $\frac{l_{\mathrm{x}}}{x}$ diminue avec $x$, il résulte des remarques ci-dessus qu'il faut essayer un $x$ plus petit.

d'où :

$$
x=20 \quad l_{\mathrm{x}}=20,0167 \quad l_{2 \mathrm{x}}=40,1345
$$

$$
\left(\frac{l_{x}}{x}\right)^{2}=1,00177 \quad\left(\frac{x}{l_{x}}\right)^{2}=1,99832 \quad \frac{l_{x}}{2 x}=1,003.36
$$

Portant dans les deux membres, on trouve:

$$
20[8.35+115793-116497] \text { et } 614,7 x+635,735
$$

La différence entre le premier et le deuxième est : 1380.

Comme, par suite des remarques ci-dessus, il faut encore diminuer $x$, j'essaye :

d'où :

$$
x=14 \quad l_{\mathrm{x}}=14,0058 \quad \lg _{\mathrm{x}}=28,0461
$$

$$
\left(\frac{l_{x}}{x}\right)^{2}=1,00050 \quad\left(\frac{x}{l_{x}}\right)^{2}=0,949116 \quad \frac{l_{x}}{2}=1,001646
$$

Portant ces valeurs dans les deux membres :

$$
14[835,25+115695,716-116,4.9)] \text { et } 614,78+614,679
$$

Le premier membre a comme valeur : 1140,54 ; le second: 1239,46 la différence cntre le premier et le deuxième est donc négative; elle est;

$$
\text { - } 98,93
$$

Ces trois essais suffisent pour le tracé dont il a déjá élé question dans l'exemple du chapitre précédent; il donne comme solution :

d'où :

$$
x_{0}=14,7
$$

donc :

$$
\mathrm{T}_{11}=\frac{184,365}{29,4}=-66,27
$$

$$
m=\frac{\mathrm{T}_{0}}{\pi}=1628 \mathrm{~m}
$$

alors que le module de la chaînetle initiale était 1721 mètres. Par suite de la faible différence entre ces deux modules et vu la petitesse de la portée, la question du terrain ne se pose pas icj.

e) Pour avoir la tension de pose à une température quelconque $\theta$, il faudrait ecrire :

$$
\begin{gathered}
L_{0}=170,470 \frac{1+x 0}{1+10 x} \\
L=L_{0}\left(1+i N_{n}\right)
\end{gathered}
$$


c'est-it-dire :

$$
\begin{aligned}
& 170,472 \cdot \frac{1+x 0}{1+10 x}= \\
& \ldots=\frac{\sqrt{144,5^{2}+\left(2 l_{x} \frac{170}{2 x}\right)^{2}}}{1+\lambda \frac{184,365}{4 x \sqrt{1+4,45}+\left(2 l_{x} \frac{170}{2 x}\right)^{2}}\left[170+l_{2 \times} \cdot \frac{171}{2 x}\right.} \\
& \cdots\left(1+\frac{11,10}{2\left(l_{\mathrm{x}} \frac{170}{2 x}\right)^{2}}\right)
\end{aligned}
$$

c'est-à-dire une équation de la forme :

$$
\frac{\sqrt{p+q\left(\frac{l_{x}}{x}\right)^{2}}+\frac{1}{2}\left[1+\frac{l_{x}}{g x}\left[1+v\left(\frac{x}{l_{x}}\right)^{2}\right]\right]}{p+q\left(\frac{l_{x}}{x}\right)^{2}}=\frac{\mathrm{A}}{\mathrm{B}+c \theta}
$$

Le premier membre est une fonction $z=f(x)$, le deuxième $z^{\prime}=f(9)$. On construira séparément ces deux courbes (fig. 13).

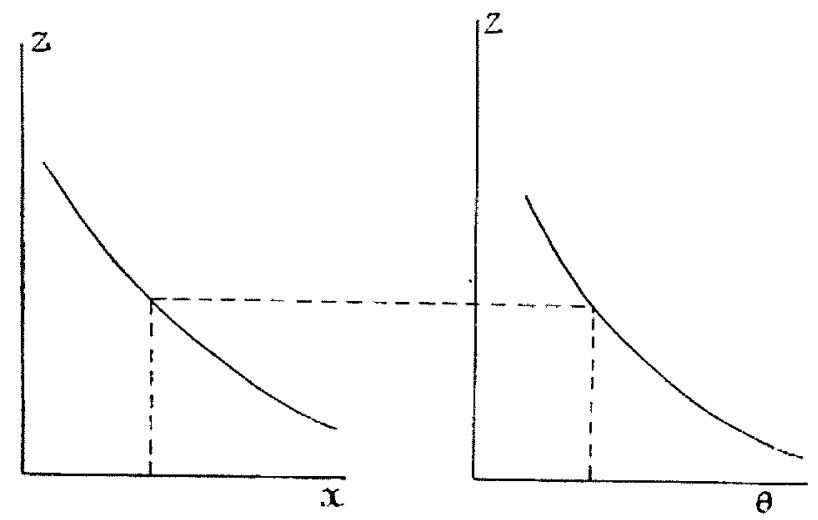

Fig. 13

Chaque horizontale donnera l' $x$ correspondant au de pose On en déduira la tension horizontale de pose puisque :

$$
\mathrm{T}_{\mathrm{i}}=\frac{184,365}{x}
$$

Le rapport de similitude avec la chan̂elle auxiliaire $\mathrm{P}$ étant

$$
\frac{T_{0}}{\mathrm{R}}=\frac{1}{j},
$$

Ia longueur de la corde du segment semblable de cette derniere est :

$$
\lambda \sqrt{14,45^{2}+170^{2}}=a b
$$

On pourra hacilement placer $a b$ sur le graphique R puisqu'on commât sa direction et sa longueur. Lordomne supérieure $y_{\mathrm{m}}$ domne la tension de pose an point datlache:

$$
\mathrm{T}==\left(y_{\mathrm{m}}+\mathrm{R}\right) \frac{1}{\lambda} \cdot \pi
$$

Si on veut connaître celle tension avec une exactilude parfaite, on la calculera au moyen de l'équation (11) oid

$$
2 d=170 \quad m_{\mathrm{p}}=\frac{\mathrm{T}_{0}}{\tau} \quad \text { II }=1,4,45 \quad \pi_{\mathrm{p}}=\pi
$$

f) Il faut aussi virifier que la tension au point le plus bas n'a pas une composante opposce au sens de la pesanteur, c'est-idire que le point le plus bas de la chaînette, dont fail partic le câble reste tonjours entre les deux verticales des points d'allache. Si cette condition est réalisée pour le càble, elle le sera aussi pour $a b$ sur la chainette graphique $R$; le controble en est donc facile graphiquement. Si on veut le vérilier algébriquement on opèrera ainsi :

Pour chaque $x$, el par suite chaque ' $T_{0}$, léqualion (11) donne $\mathrm{T}$; le rapport de similitude élant $\frac{170}{2 x}$, l'y du point de la chainelle " $r$ n correspondant au point d'altache supérieur risulle de :

$$
\frac{y_{2}+r}{\frac{T}{\pi}}=\frac{2 x}{170}
$$

les tables de a $r$ donnent l' $x_{2}$ correspondant à cet $y_{2}$.

Comme :

$$
x_{2}-x=x_{1}
$$

on voil immédiatement si le point de la chaînetle " $r$ " correspondant au point d'attache le plus bas est à droite ou à gauche de son axe de symétrie ce qui résout la question.

Remarque. - Les modules d'équilibre des câbles aluminiumacier étant compris entre 1.000 et 2.000 , il sera préférable de calculer les tables de base " $l x$ " ... etc..., pour une chaînette de référence $r=1.000$ mètres. De celte manière, le rapport de similitude sera compris entre 1 et $\frac{1}{2}$, et, les crreurs des tables, au lieu d'ètre multipliées, comme dans les calculs précédents, par un coefficient voisin de 5, le serait par un coefficient beaucoup plus faible.

N. DEWulF 